\title{
Emergence of Fermi Pockets in a New Excitonic Charge-Density-Wave Melted Superconductor
}

\author{
D. Qian, ${ }^{1}$ D. Hsieh, ${ }^{1}$ L. Wray, ${ }^{1}$ E. Morosan, ${ }^{2}$ N. L. Wang, ${ }^{3}$ Y. Xia, ${ }^{1}$ R. J. Cava, ${ }^{2}$ and M. Z. Hasan ${ }^{1,4, *}$ \\ ${ }^{1}$ Department of Physics, Joseph Henry Laboratories of Physics, Princeton University, Princeton, New Jersey 08544, USA \\ ${ }^{2}$ Department of Chemistry, Princeton University, Princeton, New Jersey 08544, USA \\ ${ }^{3}$ Institute of Physics, Chinese Academy of Sciences, Beijing 100080, China \\ ${ }^{4}$ Princeton Center for Complex Materials, School of Engineering, Princeton University, Princeton, New Jersey 08544, USA
}

(Received 5 December 2006; published 16 March 2007)

\begin{abstract}
A superconducting state $\left(T_{c} \sim 4.2 \mathrm{~K}\right)$ has very recently been observed upon successful doping of the charge-density-wave (CDW) ordered triangular lattice $\mathrm{TiSe}_{2}$, with copper. Using state-of-the-art photoemission spectroscopy we identify, for the first time, momentum-space locations of doped electrons that form the Fermi sea of the superconductor. With doping, we find that kinematic nesting volume increases, whereas coherence of the CDW collective order sharply drops. In superconducting doping, as chemical potential rises, we observe the emergence of a large density of states in the form of a narrow electron pocket near the $L$ point of the Brillouin zone with $d$-like character. The $k$-space spectral evolution directly demonstrates, for the first time, that the CDW order parameter microscopically competes with superconductivity in the same band.
\end{abstract}

PACS numbers: 74.20.Mn, 71.45.Lr, 74.50.+r, 74.72. $-\mathrm{h}$

Charge-density waves (CDW) and superconductivity are two of the most fundamental collective quantum phenomena in solids. The competition between the two is one of the central issues of condensed matter physics [16]. A microscopic study of this competition is often difficult for two reasons: one, most systems involve some form of intervening phases such as magnetic order observed in the parent state of cuprate [3] or cobaltate [4] superconductors; two, there lacks systematically tunable electronic parameters compatible with microscopic $k$-space imaging techniques such as angle-resolved photoemission spectroscopy (ARPES). Until very recently, no system existed where controlled chemical tuning allowed this competition to be studied in microscopic detail. Recent investigations have shown that upon controlled intercalation of $\mathrm{TiSe}_{2}$ with copper $\left(\mathrm{Cu}_{x} \mathrm{TiSe}_{2}\right)$ one can systematically tune between a CDW state and a novel superconducting state which emerges near $x=0.04$, with a maximum $T_{c}$ of $4.15 \mathrm{~K}$ reached near $x=0.08$ [1]. A quantum melting of CDW order was also observed recently in the parent compound by pressure [7]. $\mathrm{Cu}_{x} \mathrm{TiSe}_{2}$ thus provides a rare opportunity to systematically investigate quantum (zero or low temperature) phase transition from a CDW ordered state to superconductivity.

The parent compound $\mathrm{TiSe}_{2}$ has been known since the 1960s and has been extensively studied [8-14] due to the unconventional nature of the CDW state. In this Letter, we use high resolution ARPES to image the momentum-space evolution of doped electrons as they evolve from the undoped CDW ordered phase to an optimal superconductor by copper doping for the first time. A detailed knowledge of momentum-space distribution of electronic states is critically important in developing a theory for the superconducting order parameter $(s, p$, or $d$ wave) realized in this novel materials class. Based on the emergent Fermi surface topology and momentum-space distinctions of par- ticipant electrons in each phase we directly show that the superconducting phase emerges upon the gradual loss of coherence of the CDW state on the same site. Thus the two states compete in a microscopic manner and not due to phase separation or other extrinsic effects as observed in other known systems. In the superconducting doping we observe the emergence of a large density of states in the form of an extremely narrow electron pocket of about $100 \mathrm{meV}$ bandwidth leading to a nontrivial Fermi surface topology.

Undoped $\mathrm{TiSe}_{2}$ is a layered material with trigonal structure and hexagonal Brillouin zone. From previous high or finite temperature photoemission studies of undoped material [10], $\mathrm{TiSe}_{2}$ is believed to be either a semimetal or a very small gap semiconductor. It undergoes a $(2 \times 2 \times 2)$ structural transition below the CDW transition temperature $T_{c}=220 \mathrm{~K}$. Upon doping, the resistivity anomaly near $200 \mathrm{~K}$ is suppressed and samples become more metallic-
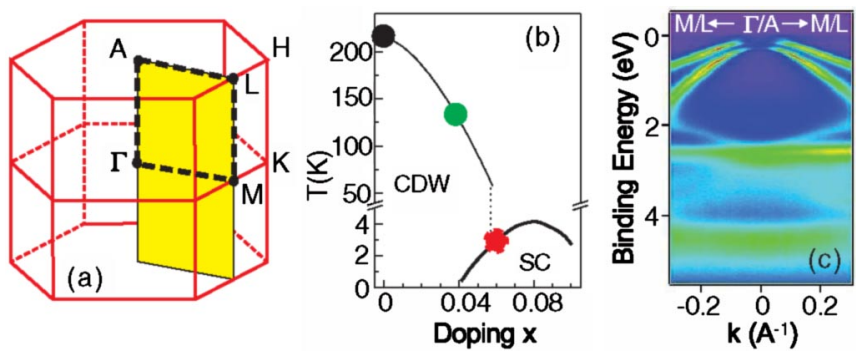

FIG. 1 (color online). Sample characterization: (a) Brillouin zone layout of $\mathrm{Cu}_{x} \mathrm{TiSe}_{2}$ where $M\left(\frac{1}{2}, 0,0\right), K\left(\frac{1}{3}, \frac{1}{3}, 0\right), L\left(\frac{1}{2}, 0, \frac{1}{2}\right)$, $H\left(\frac{1}{3}, \frac{1}{3}, \frac{1}{2}\right)$, and $A\left(0,0, \frac{1}{2}\right)$. (b) Doping-temperature phase diagram suggests $\mathrm{CDW}$ is a competing phase for superconductivity. (c) High resolution valence band profile of $\mathrm{Cu}_{x} \mathrm{TiSe}_{2}$ along the $M / L-\Gamma / A-M / L$ momentum-space cut measured at $20 \mathrm{~K}$. The top two bands are the $\Gamma^{3}\left(A^{3-}\right)$ and $\Gamma^{2-}$ bands (in agreement with LDA calculations [16]). 
like and eventually superconduct at low temperatures as measured in powder samples [1]. Very recently, high quality single crystal samples were grown [15] making $k$-resolved measurements possible. ARPES measurements were carried out at beam line ALS-12.0.1 at the Advanced Light Source and SRC-U-NIM beam line at the Synchrotron Radiation Center. The data were collected with 13 to $79 \mathrm{eV}$ photons with better than 10 to $25 \mathrm{meV}$ energy resolution and an angular resolution better than $1 \%$ of the Brillouin zone using Scienta analyzers with chamber pressure better than $8 \times 10^{-11}$ torr. Photon energies of 13 and $56 \mathrm{eV}$ were used for measurements near the $\Gamma$ point, whereas 19 and $78 \mathrm{eV}$ photons were used for data collection near the $L$ point. Cleaving the samples in situ at 20 or $250 \mathrm{~K}$ resulted in shiny flat surfaces characterized by diffraction being clean and well ordered with the same symmetry as the bulk. The measured valence band (Fig. 1) was found to be in good agreement with localdensity approximation (LDA) band theory [16] irrespective of the cleaving temperature.

The low temperature evolution of momentum-resolved electronic structure of $\mathrm{TiSe}_{2}$ with copper doping is shown in Fig. 2. A spectacular change of low-energy electronic structure is observed with systematic doping. The emergence of a pocketlike band near the $L$ point is very prominent at low temperatures. With copper doping the density of states near the $L$ point increases systematically while the states below $0.2 \mathrm{eV}$ are seen to be systematically suppressed. At low temperatures, the states below $0.2 \mathrm{eV}$ near the $L$ point are due to the folding of bands from the $\Gamma$ point via the $2 \times 2 \times 2$ reconstruction, which is a consequence of the CDW order. With copper doping, we observe a weakening of folding strengths in $x=0.04$ and $x=0.06$ samples. In fact, in the $x=0.06$ samples folding is extremely weak suggesting a short range nature or near absence of the CDW order. Figure 2 (bottom row) shows evolution near the $\Gamma$ point. A systematic sinking of the band multiplet is observed to occur with doping. This is accompanied by some finite growth of intensity near the Fermi level over an extended $k$ range. However, no clear quasiparticle crossing is resolved in the energy dispersion curves near the $\Gamma$ point. Selected energy dispersion curves for the temperature evolution of electronic states near $k=$ $L+0.1 \AA^{-1}$ with doping are shown in Fig. 3. The prominent peak at $0.25 \mathrm{eV}$ in $x=0$ at $T=20 \mathrm{~K}$ is due to band folding and constitutes the key spectral signature of CDW order. This peak grows in intensity and moves to higher binding energies with decreasing temperature. With increasing copper doping the intensity of the $0.25 \mathrm{eV}$ peak drops, while the intensity or the density of states near the Fermi level grows dramatically.

Above the CDW transition temperature (for $T>$ $220 \mathrm{~K}$ ), in the doped system, we find that chemical potential is near the midpoint of the leading edge of the quasiparticle peak, which is a clear indication that the doped compounds are metals (Figs. 2-4). In contrast, the location of chemical potential in the undoped system is at the peak (highest intensity), suggesting it is either the thermal tail signal from a band that barely grazes the Fermi level. Therefore, it is not directly resolved within finite temperature ARPES data. However, for data taken at sufficiently low temperatures $(T \sim 20 \mathrm{~K})$ the end compound exhibits a metalliclike component with low carrier density. This could be intrinsic or due to excess titanium in the bulk. If not due to excess titanium this would suggest that the high temperature phase is also metallic due to the charge neutrality and particle-hole symmetry constraint. The high temperature evolution of electronic structure with copper doping is shown in Fig. 4. With doping, spectral weight is transferred toward the Fermi level; however, no clear (a) $\mathrm{Cu} x=0$

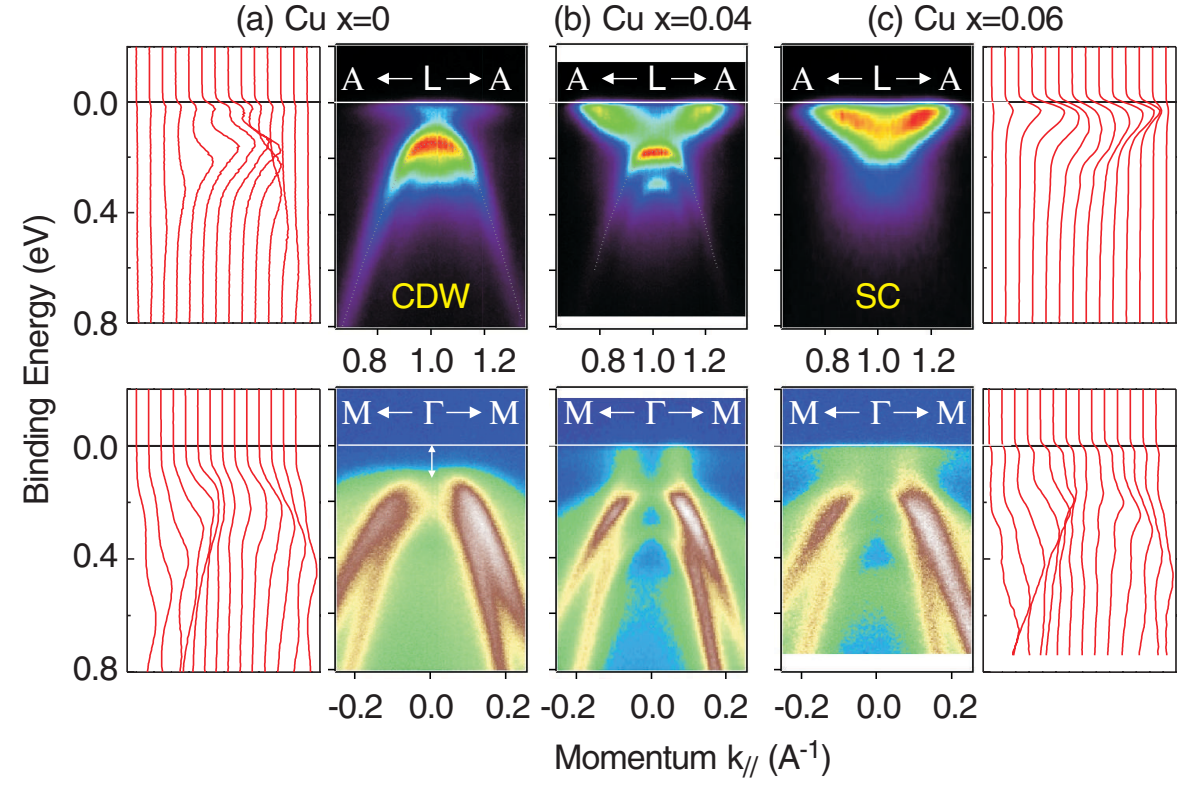

$$
\mathrm{T}=20 \mathrm{~K}
$$

(b) $\mathrm{Cu} x=0.04$

FIG. 2 (color online). CDW state melts near superconducting doping: low temperature $\left(T \ll T_{\mathrm{CDW}}\right.$ at $\left.x=0\right)$ doping evolution of bands in the vicinity of the $L$ point (top row) and $\Gamma$ points (bottom row). With increasing doping the intensity of the folded band decreases and spectral weight concentrates near the $L$ points in the form of an electron pocket with bandwidth about $0.1 \mathrm{eV}$. Bottom row: with increasing doping spectral weight near the $M-\Gamma-M$ cuts continues to shift to lower energies and deeperlying bands tend to split further from each other. 


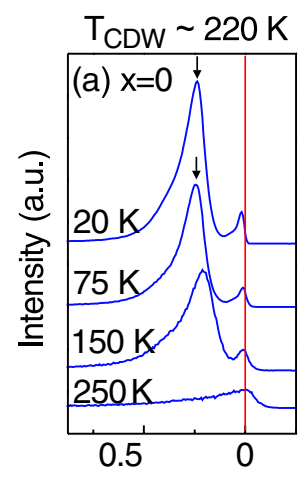

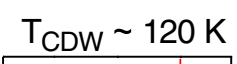

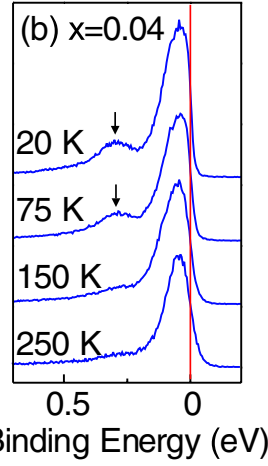

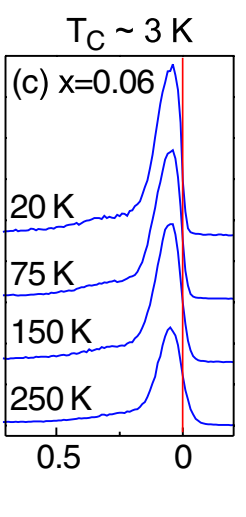

FIG. 3 (color online). Temperature evolution of spectral function with doping: energy distribution curves for $k=$ $L+0.1 \AA^{-1}$ are shown for $x=0,0.04$, and 0.06 dopings. The peak at $0.25 \mathrm{eV}$ in $x=0$ at $T=20 \mathrm{~K}$ is due to band folding. With increasing doping the intensity of the $0.25 \mathrm{eV}$ peak drops while the quasiparticle peak grows dramatically.

crossing is resolved near the $\Gamma$ point in our study. In contrast, a pocketlike band near the $L$ point is found to sink below the Fermi level with doping (bottom row). In band structure calculations, this band derives from the Ti-3d-like states. A fit to the energy-distribution curves at $k=L$ for an $x=0$ sample suggests that the band barely touches the Fermi level or slightly above the Fermi level. With copper doping we observe a systematic sinking of this band reaching about $0.1 \mathrm{eV}$ in bandwidth near $x=0.06$ doping. Since the size of the pocket grows with increasing doping we conclude an electron doping (as opposed to hole doping) scenario with the intercalation of copper. This is consistent with bulk measurements such as the negative sign of thermopower $(S / T)$ [1].

We have carried out a detailed measurement of electron distribution $n(k)$ in the $A-L$ plane in all metallic samples of $\mathrm{Cu}_{x} \mathrm{TiSe}_{2}$. The presented data in Fig. 5 are shown with an energy integration window of about $20 \mathrm{meV}$ within the Fermi level and were taken in the first quadrant, then symmetrized over the Brillouin zone. This is checked by direct measurements in other quadrants and found to be consistent with the symmetrization procedure apart from intensity variations due to changing matrix elements. Large intensities in the form of ellipses are observed at the midpoints of the hexagonal arms. In the superconducting doping samples $\mathrm{Cu}_{0.06} \mathrm{TiSe}_{2}$, these zone-boundary ellipses resolve as electronlike pockets (Fig. 5) which originate from the sinking band near the $L$ point in Fig. 4.

With increasing doping the gap closes and low-energy states cross the Fermi level. This leads to the emergence of kinematic nesting instabilities (non- $2 \times 2 \times 2$ type) along $A-L$ and $A-H$. Our data show that the CDW state loses long-range coherence with doping but no new superstructure is observed as can be seen by band folding. Such loss of coherence is quite evident from decreasing intensity of the $0.25 \mathrm{eV}$ feature near the $L$ point (Fig. 3). Within band theory no nesting is expected to generate a $2 \times 2 \times 2$ commensurate superstructure for the electron distribution in the $x=0$ compound [16]. With copper doping we find that the electron pocket near the $L$ point continues to grow, thereby increasing the phase space for nesting, but no new band folding is observed and the strengths of $2 \times 2 \times 2$ folding continue to drop, ruling out nesting as a mechanism for CDW in the doped system.

Following the theoretical suggestion of Kohn [17], an electronic (excitoniclike) mechanism has recently been proposed to account for the $\mathrm{CDW}$ order of the parent compound [13]. If this scenario is correct, one would expect the electron-hole coupling necessary to generate a gap to decrease in the doped systems since the CDW loses long-range coherence with doping. In this scenario, evidence for the loss of coherence comes from the fact that the bottom of the pocket band at the $L$ point [Fig. 2(c) (top panel)] is not as flat as seen in the undoped compound previously. It is unclear if this scenario is supported in doped systems since no clear recovery of the paraboliclike shape is evident in the doped system; however, the increasing lack of particle-hole symmetry (Fig. 2) away from the undoped compound destabilizes the exciton formation which requires this symmetry by raising the chemical potential (Fig 4).

It is well known that increasing density of states (DOS) at the Fermi level $D\left(E_{f}\right)$ enhances superconducting pairing correlations in a system. The structurally analogous novel
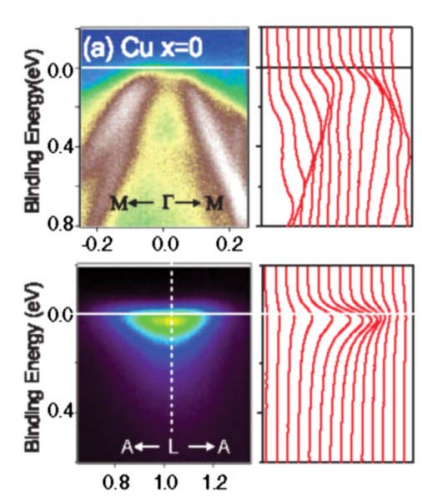

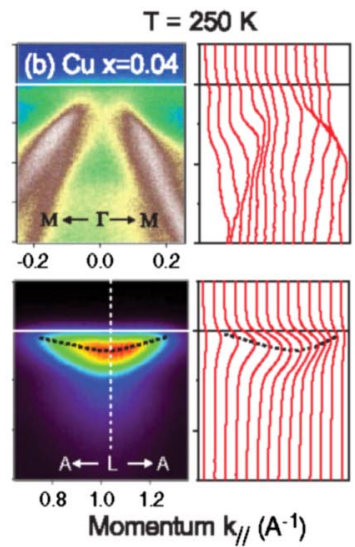

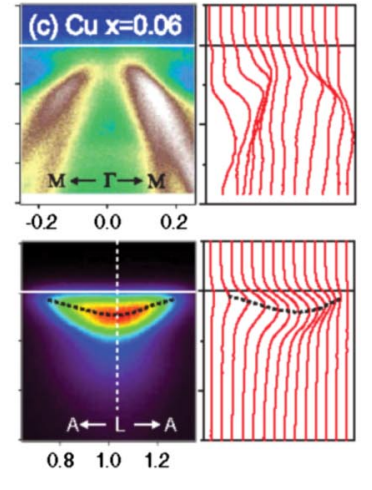

FIG. 4 (color online). Rise of chemical potential with doping: Electron pockets emerge and grow with doping: high temperature $\left(T \gg T_{\mathrm{CDW}}\right)$ band evolution in the vicinity of the $L$ point. With increasing doping the Fermi level rises up. At the $\Gamma$ point this is seen as a lowering of the Se $4 p$ bands [(a)-(c), top row] and the emergence of low-energy states. At the $L$ point, doping gives rise to an electron pocket with a bandwidth of about $0.1 \mathrm{eV}$. The $L$ pocket grows with increasing doping. 


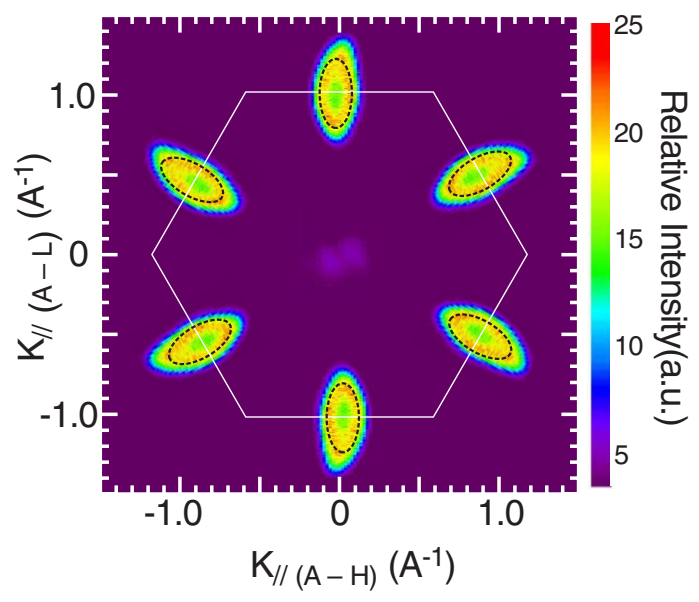

FIG. 5 (color online). Electron distribution of superconductor $\mathrm{Cu}_{0.06} \mathrm{TiSe}_{2}$ : with increasing copper doping, electrons near the $L$ point form a narrow pocket and generate a sixfold symmetric Fermi contour. A finite but weak intensity is also observed at the $\Gamma / A$ point. The presented summary of data above is measured in the first quadrant at $T=20 \mathrm{~K}$ then symmetrized over the Brillouin zone.

superconductor $\mathrm{Na}_{x} \mathrm{CoO}_{2}$ exhibits enhancement of $D\left(E_{f}\right)$ with $\mathrm{Na}$ doping in the presence of hydration [4]. It is known that $D\left(E_{f}\right)$ also increases in the Fe-doped systems $\mathrm{Fe}_{x} \mathrm{TiSe}_{2}$ and $\mathrm{Fe}_{x} \mathrm{TiTe}_{2}[9,14]$, however no superconductivity is observed in any of these systems. It is likely that the magnetic moments in the $\mathrm{Fe}_{x} \mathrm{Ti}(\mathrm{Se} / \mathrm{Te})_{2}$ are strong enough to suppress superconductivity via nonunitary interactions (renormalizing $T_{c}$ to zero). In $\mathrm{Cu}_{x} \mathrm{TiSe}_{2}$, copper is monovalent $\left(\mathrm{Cu}^{+1}\right)$ and nonmagnetic $\left(3 d^{10}\right.$ and $\left.S=0\right)$. Therefore its main role is to donate an electron to the TiSe layer which then delocalizes. We have observed in this work that this electron mostly enters the parent band around the $L$ point that has $d$-like character which exhibits weak dispersion (less than $100 \mathrm{meV}$ ). Therefore, the overall effect of nonmagnetic copper doping is to raise the $d$-like density of states $D\left(E_{f}\right)$ at the Fermi level. Such increases in $D\left(E_{f}\right)$ lead to a rise in $T_{c}$ in many systems, as expected in the BCS-Eliashberg scenario: $k_{B} T_{c}=$ $\hbar \omega \exp \{-1 /[D(E f) V]\}$.

Finally, we note that the Fermi velocity $\left(v_{F}\right)$ of the $L$-point electron pocket is about $0.4 \pm 0.1 \mathrm{eV} \AA$ [see $E$ vs $k$ in Fig. 4(c)]. This allows us to estimate the expected coherence length in the BCS-Eliashberg $s$-wave scenario: $\xi \sim 0.19 \frac{\hbar v_{F}}{k_{B} T_{c}} \sim 29 \mathrm{~nm}$ under the assumption that this band is the Fermi sea for the superconducting pairing in this compound. This value is remarkably close to the value of $22 \mathrm{~nm}$ estimated from $H_{c 2}$ measurements [15] for $x=0.07$ samples, which provides further support for our identification of this $L$-pocket electron taking part in superconducting pairing in this compound. It is known that the CDW phase of the $x=0$ parent compound is associated with considerable softening of an $L$ phonon [18]. It is possible that the $L$ phonon plays a key role in the superconducting mechanism. Our preliminary results do not necessarily rule out an electronic mechanism of superconductivity.

In conclusion, we have reported the first measurement of low-lying states and Fermi surface topology of the copper doped CDW superconductor. Our results show that an electronlike pocket near the $L$ point continues to grow upon doping into the superconducting phase. A systematic loss of long-range coherence of the CDW state is observed at low temperatures via the loss of spectral intensity from folded bands with increasing doping. This demonstrates that the CDW microscopically competes with superconductivity. Momentum-space distribution of electronic states is crucial in developing a microscopic theory/mechanism for the superconducting order parameter realized in this novel materials class. The reported evolution of overall electronic structure with copper doping will be further helpful in formulating a theory of CDWsupercondctivity competition in general.

We acknowledge discussions with D. A. Huse, P. W. Anderson, S. Sondhi, S. Sachdev, N. P. Ong, A. Fedorov, and $\mathrm{W}$. Ku. This work is supported by DOE Grant No. DEFG-02-05ER46200. R. J. C. and M.Z. H. acknowledge support through NSF (No. DMR-0213706). A.L.S. is supported by No. DOE/DE-AC02-05CH11231; SRC/UW is supported by No. NSF/DMR-0537588.

*Electronic address: mzhasan@princeton.edu

[1] E. Morosan et al., Nature Phys. 2, 544 (2006).

[2] S. A. Kivelson et al., Nature (London) 393, 550 (1998).

[3] P. A. Lee et al., Rev. Mod. Phys. 78, 17 (2006); S. Sachdev and S. C. Zhang, Science 295, 452 (2002); M. Imada et al., Rev. Mod. Phys. 70, 1039 (1998).

[4] K. Takada et al., Nature (London) 422, 53 (2003); M.Z. Hasan et al., Phys. Rev. Lett. 92, 246402 (2004); D. Qian et al., Phys. Rev. Lett. 96, 216405 (2006); 96, 046407 (2006); 97, 186405 (2006).

[5] L. Fang et al., Phys. Rev. B 72, 014534 (2005).

[6] D. E. Moncton et al., Phys. Rev. Lett. 34, 734 (1975).

[7] C. S. Snow et al., Phys. Rev. Lett. 91, 136402 (2003).

[8] J. A. Wilson and A. D. Yoffe, Adv. Phys. 18, 193 (1969).

[9] F. J. Di Salvo et al., Phys. Rev. B 14, 4321 (1976).

[10] R.Z. Bachrach et al., Phys. Rev. Lett. 37, 40 (1976); M. M. Traum et al., Phys. Rev. B 17, 1836 (1978); G. Margaritondo et al., Phys. Rev. B 23, 3765 (1981); O. Anderson et al., Phys. Rev. Lett. 55, 2188 (1985); N. G. Stoffel et al., Phys. Rev. B 31, 8049 (1985).

[11] Th. Pillo et al., Phys. Rev. B 61, 16213 (2000).

[12] K. Rossnagel et al., Phys. Rev. B 65, 235101 (2002).

[13] T. E. Kidd et al., Phys. Rev. Lett. 88, 226402 (2002).

[14] K. Yamazaki et al., Physica (Amsterdam) 351B, 262 (2004); X. Y. Cui et al., Phys. Rev. B 73, 085111 (2006).

[15] E. Morosan et al. [Phys. Rev. B (to be published)].

[16] A. Zunger et al., Phys. Rev. B 17, 1839 (1978).

[17] W. Kohn, Phys. Rev. Lett. 19, 439 (1967); A. W. Overhauser, Phys. Rev. Lett. 4, 415 (1960).

[18] M. Holt et al., Phys. Rev. Lett. 86, 3799 (2001). 